\title{
Deux nouvelles Parastenocaris (Copépodes, Harpacticoïdes) des Pyrénées
}

\author{
R. Rouch 1
}

Mots clés : Copepoda, Harpacticoida, espèces nouvelles, faune aquatique souterraine.

Description de Parastenocaris mertensis n. sp. et Parastenocaris fontinalis meridionalis $\mathrm{n}$. s.sp. récoltées dans le sousécoulement d'un ruisseau des Pyrénées.

Two new species of Parastenocaris (Copepoda, Harpacticoida) from the Pyrenees.

Keywords : Copepoda, Harpacticoida, new species, stygobiont fauna.

Descriptions aregiven of Parastenocaris nertensis n. sp. and Parastenocaris fontinalis meridionalis $\mathrm{n} . \mathrm{s}$. sp. from the Nert brook underflow, Ariège, France.

Le sous-écoulement du ruisseau le Nert, affluent de rive droite du Salat (Ariège), possède une riche faune interstitielle hypogée (Gourbault \& LescherMoutoué 1967, 1968). Trois espèces d'Harpacticides stygobies y ont été signalées par ces auteurs, Parapseudoleptomesochra subterranea (Chappuis 1928), Nitocrella gracilis Chappuis 1955 et Parastenocaris dianae Chappuis 1955 , qui sont des taxons relativement communs dans les eaux souterraines de l'Ariège.

Une nouvelle série de prélèvements récemment réalisés quelques mètres en amont de la station 16 définie par Gourbault et Lescher-Moutoué montre que la présence du genre Parastenocaris dans le sousécoulement du Nert ne se limite pas à une seule espèce. En effet, quatre taxons cohabitent au sein des alluvions de cette station : $P$. dianae, $P$. vandeli Rouch 1988, $P$. nertensis n. sp. et $P$. fontinalis meridionalis n. s.sp. La description de ces nouvelles espèce et sous-espèce fait l'objet de la présente note.

1. Laboratoire souterrain du CNRS, Moulis, 09200 SaintGirons, France.

\section{Parastenocaris nertensis n. sp.}

Localité et matériel : Ruisseau le Nert, commune d'Encourtiech, Ariège, France. Pompage dans le sous-écoulement par sondage tubé, le 19 décembre 1989. Matériel examiné : $5 \%$ et $90^{\circ} \sigma^{\circ}$.

Holotype : 1 o conservé dans la collection de l'auteur.

\section{DESCRIPTION}

\section{- MÃLE :}

Longueur moyenne du corps sans les soies furcales : $402 \mu \mathrm{m}$. Bord postérieur des segments du corps lisse ; segments de l'urosome sans ornementation. Opercule anal glabre (fig. 1 a). Branches furcales allongées (fig. 1 a et b), 4,4 fois plus longues que larges, avec une seule épine apicale bien développée, flanquée d'une fine soie interne; la partie ctistale du bord externe de chaque rame est biseautée et porte trois épines; l'épine dorsale, articulée à sa base, s'insère près du bord interne.

Antennule ( $f i g .1 \mathrm{c}$ ). Préhensile, formée de sept articles, avec un aesthétasque sur le quatrième article et une soie sensitive sur le septième.

Antenne (fig. $1 \mathrm{~d}$ ). Allobasis avec une épine au bord interne. Exopodite uniarticulé avec une épine 
apicale. Endopodite uniarticulé avec deux épines et deux séries de spinules au bord interne et cinq épines apicales.

Mandibule (fig. 1 e). Precoxa avec une pars incisiva et une pars molaris bidentées, une rangée de dents et une soie. Palpe uniarticulé avec deux soies apicales.

Maxillule (fig. 1 f). Arthrite de la precoxa avec trois crochets distaux et une soie subapicale. Coxa et basis avec, respectivement, une et trois épines apicales.

Maxille (fig. $1 \mathrm{~g}$ ). Syncoxa avec un seul endite portant deux soies. Basis avec un fort crochet. Endopodite représenté par deux soies.

Maxillipède (fig. $1 \mathrm{~h}$ ). Basis et premier article de l'endopodite glabres. Deuxième article de l'endopodite avec un fort crochet.

P1 (fig. 1 i). Basis avec une courte épine externe et deux rangées de spinules. Premier article de l'endopodite avec deux rangées de spinules au bord externe et deux spinules au bord interne ; deuxième article avec une courte épine médiane et deux épines apicales. Premier article de l'exopodite avec une épine entourée de spinules à l'angle distal externe ; deuxième article avec quelques spinules à l'angle distal externe ; troisième article avec quatre épines.

$\mathbf{P} 2$ (fig. 1 j). Basis avec une rangée de spinules. Endopodite digitiforme, sinueux, avec quatre spinules à son extrémité distale et deux petites spinules au bord externe. Exopodite avec une épine apicale au bord externe du premier article et trois épines apicales au troisième article.

P3 (fig. $1 \mathrm{k}$ ). Basis avec une longue épine externe et une rangée de spinules. Exopodite très élancé, mince, avec quelques spinules au bord externe, se terminant par une apophyse allongée. Le pouce, faiblęment chitinisé, ne dépasse pas la moitié de la longueur de l'apophyse. L'endopodite est représenté par une longue soie hyaline qui s'insère au bord interne du basis.

P4 (fig. 1 1). Basis avec une épine externe et une rangée de spinules. L'endopodite assez large dans sa partie proximale, devient ensuite digitiforme à la suite d'une forte échancrure de son bord interne ; cette partie digitiforme est très faiblement barbelée du côté externe ; l'endopodite est armé de trois crochets à sa base. Exopodite avec une épine apicale au bord externe du premier article et deux épines apicales au troisième article

P5 (fig. $1 \mathrm{~m}$ ). Faiblement développée, elle se présente sous la forme d'une lamelle rectangulaire armée de quatre épines à son bord distal.

\section{- Femelle :}

Longueur moyenne du corps sans les soies furcales : $390 \mu \mathrm{m}$. Ornementation du corps, branches furcales et opercule anal comme chęz le mậlẹ.

Antennule (fig. 2 a). 7 articles. Un aesthétasque sur le quatrième et le septième article.

Antenne, mandibule, maxillule, maxille, maxillipède, $\mathrm{P} 1$ (fig. 2 b), $\mathrm{P} 2$ (fig. 2 c) et exopodite $\mathrm{P} 4$ (fig. 2e) sans différences notables avec les appendices correspondants du mâle.

P3 (fig. 2 d). Basis avec une épine externe et une rangée de spinules. Exopodite biarticulé avec une épine distale externe au premier article et deux épines apicales au second. Endopodite biseauté dans sa partie distale externe avec deux petites spinules.

Endopodite P4 (fig. 2 e). Digitiforme, il se retrécit dans sa partie distale pour se terminer en pointe. Trois spinules internes et une externe s'insèrent au niveau de ce rétrécissement. L'endopodite est flanqué de trois épines à sa base.

P5 (fig. $2 \mathrm{f}$ et $\mathrm{g}$ ). Comme chez le mâle, la P5 est peu développée. De forme rectangulaire, elle est armée de quatre épines à son bord distal.

\section{AFFINITÉS}

La forme de l'endopodite P4 du mâle de cette espèce ainsi que la présence d'épines à sa base conduisent à placer $P$. nertensis $\mathrm{n}$. sp. dans le groupe minuta (Lang 1948). Lang a reconnu plusieurs sousgroupes dans cet ensemble dont celui constitué, à l'époque, par $P$. phreatica Chappuis 1936, $P$. stammeri Chappuis 1937, $P$. orcina Chappuis 1938 et $P$. minuta Chappuis 1925. En fait, il est peu probable que $P$. minuta puisse être rattachée à ce sous-groupe puisque, selon Chappuis (1925 p. 182), l'endopodite P4 du mâle de cette espèce est « digitiforme, barbelé à son extrémité du côté externe " alors que chez les trois autres espèces cet endopodite se présente, globalement, sous la forme d'une lamelle fotiacée avec plusieurs ramifications plus ou moins développées. 


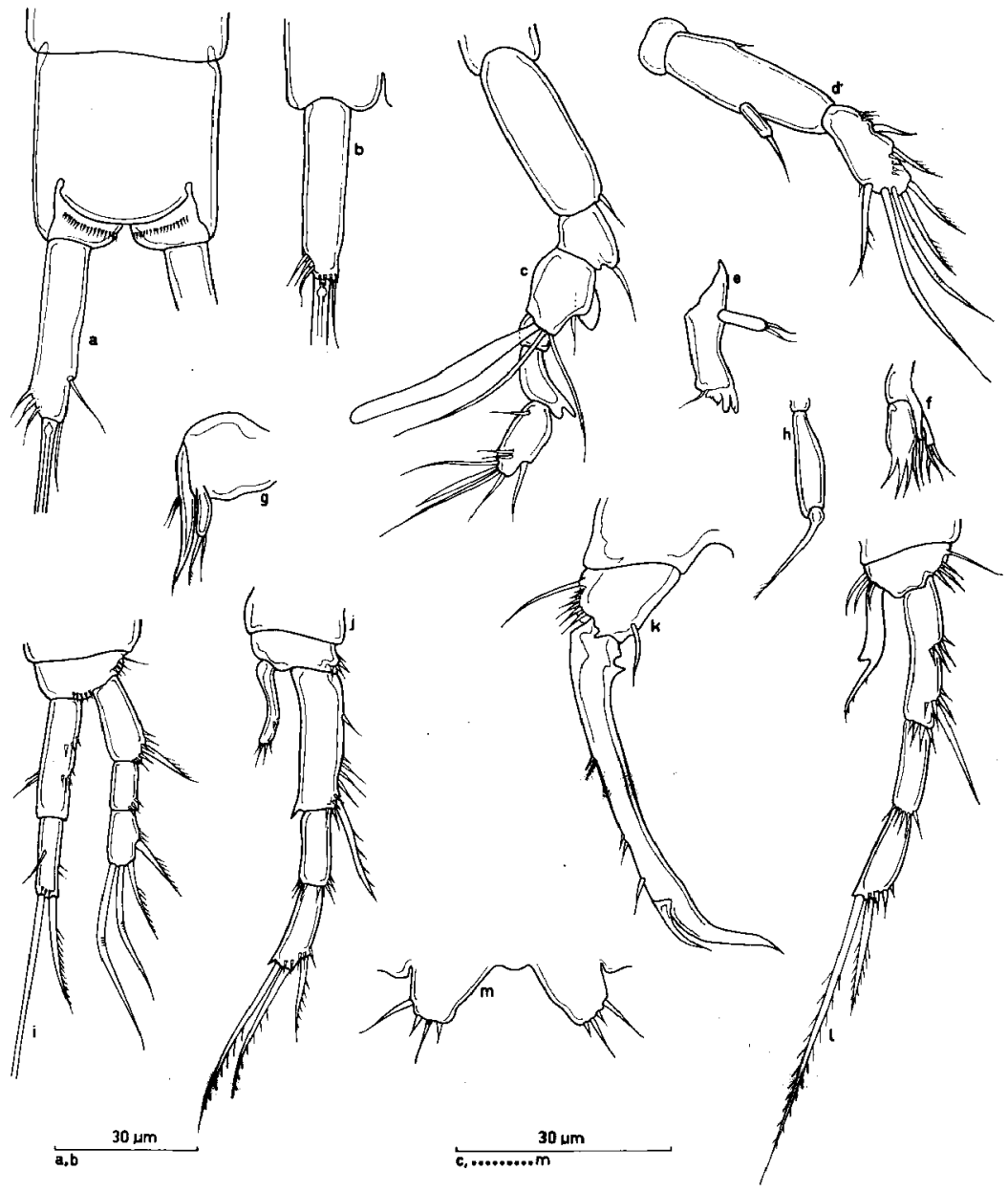

Fig. 1. Parastenocaris nertensis n. sp., $\propto$.

$a:$ dernier segment abdominal et rame furcale, vue dorsale $; b:$ rame furcale, vue ventrale $; c:$ antennule $; d:$ antenne ; $\mathrm{e}$ : mandibule ; $\mathrm{f}$ : maxillule ; $\mathrm{g}$ : maxille $\mathrm{h}:$ maxillipède $; \mathrm{i}: \mathrm{P1} ; \mathrm{j}: \mathrm{P} 2 ; \mathrm{k}: \mathrm{P} 3 ; 1: \mathrm{P4} ; \mathrm{m}: \mathrm{P}$. 


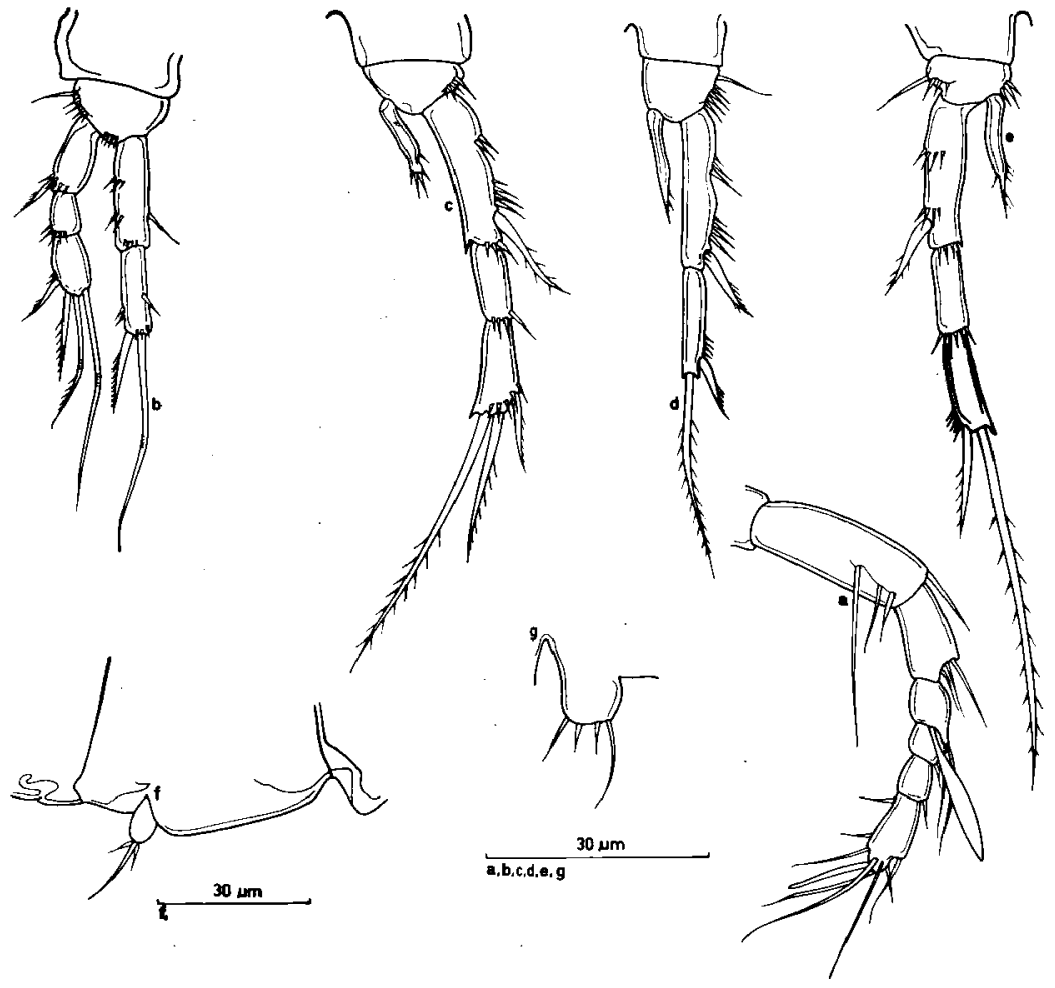

Fig. 2. Parastenocaris nertensis n. sp., $Q$.

a : antennule ; b : P1 ; c: P2; d : P3 ; e : P4; f : P5 vue latérale ; $g$ : P5 vue ventrale.

A l'heure actuelle, outre les trois formes mentionnées ci-dessus, ce sous-groupe comprend les espèces suivantes: $P$. acherusia Noodt $1955, P$. balcanica Petkovski 1959, P. stammeri gallicus Chappuis \& Rouch 1959, P. dentulatus Chappuis \& Rouch $1959, P$. andalusica Enckell 1965, $P$. amyclaea Cottarelli 1969, $P$. hera Cottarelli 1969, $P$. pasquinii
Cottarelli 1972, $P$. stellae Cottarelli et al. 1981, $P$. numidiensis Rouch 1987, P. trinacriae Pesce et al. 1988.

La structure la plus simple de l'endopodite P4 du mâle de ces espèces, sans prendre en compte les épines situées à sa base, est celle où le nombre d'excroissances de cet article est égal à deux : c'est le 
cas de $P$. orcina, $P$. balcanica et $P$. trinacriae. Ces digitations passent ensuite au nombre de trois chez $P$. phreatica, $P$. stammeri, $P$. acherusia, $P$. andalusica, $P$. hera, $P$. stellae et $P$. numidiensis et atteignent enfin le nombre de quatre chez $P$. amyclaea et $P$. pasquinii. $P$. dentulatus s'écarte de cet ensemble puisque ce sont deux éléments foliacés indépendants qui participent à la constitution de l'endopodite $\mathrm{P} 4$ du mâle.

Par sa structure de la partie foliacée de l'endopodite $\mathrm{P} 4$ du mâle, l'espèce du Nert est voisine de $P$. orcina, $P$. balcanica et $P$. trinacriae ; elle en diffère par l'ornementation beaucoup plus réduite de la partie digitiforme de cet endopodite. $P$. balcanica et $P$. orcina avec, respectivement, deux et quatre épines à la base de l'endopodite $\mathbf{P 4}$ du mâle se distinguent de $P$. nertensis qui en possède trois comme $P$. trinacriae; tout efois, chez cette dernière, l'épine médiane est fortement développéc.

L'exopodite de la $\mathrm{P} 3$ du mâle de $P$. nertensis, très élancé, notamment dans la partie qui s'étend du basis à l'insertion du pouce, diffère de celui, plus court, des trois autres espèces. Le caractère distinctif le plus apparent de $P$. nertensis est constitué par sa P5 : chez le mâle comme chez la femelle, il s'agit d'une petite lamelle rectangulaire et non pas taillée en biseau et se terminant en pointe comme c'est le cas chez les trois autres espèces.

Le rapport longueur/largeur des rames caudales de ces quatre espèces est également différent : sa valeur est de $3 \mathrm{chez} P$. balcanica, de $3,6 \mathrm{chez} P$. $t r i$ nacriae, de 6 chez $P$. orcina in Chappuis $(4,8$ in Cottarelli \& Drigo 1972), de 4,4 chez $P$. nertensis.

L'ornementation de ces rames caudales est également différente.

En outre, l'endopodite P4 de la femelle de $P$. nertensis, présente trois crochets à sa base, comme chez le mâle, ornementation qui semble absente chez la femelle de $P$. orcina et $P$, trinacriae.

\section{Parastenocaris fontinalis meridionalis}

\section{n. s.sp.}

Localité et matériel : Ruisseau le Nert, communc d'Encourtiech, Ariège, France. Pompage dans le sous-écoulement par sondage tubé, le 19 décembre 1989. Matériel examiné : 4 \& \& et $7 \sigma^{\circ} \sigma^{*}$

Holotype : $1 \sigma^{*}$ conservé dans la collection de l'auteur.

\section{DESCRIPTION}

\section{- MÁle :}

Longueur moyenne du corps sans les soies furcales : $431 \mu^{\mathrm{m}}$. Bord postérieur des segments du corps lisse. Urosome avec une rangée de sept spinules sur la face ventrale et à la base des branches furcales. Opercule anal arrondi et glabre (fig. 3 a). Branches furcales 3,6 fois plus longues que larges avec trois épines apicales dont la médiane est la plus développée, une épine flanquée de deux spinules sur le bord externe et une épine dorsale géniculee (fig. 3 a et b).

Antennule, antenne, mandibule, maxillule, maxille et maxillipède (voir fig, $3 \mathrm{c}, \mathrm{d}, \mathrm{e}, \mathrm{f}, \mathrm{g}, \mathrm{h}$ ).

P1 (fig. 4 a). Basis avec une courte épine et une rangée de spinules. Premier article de l'endopodite avec deux rangées de spinules au bord externe et une épine au bord interne; deuxième article avec deux épines apicales. Exopodite avec une épine à l'angle distal externe du premier article et quatre épines au troisième article.

$\mathrm{P} 2$ (fig. 4 b). Basis une rangéc de spinules. Endopodite flexueux avec deux spinules au bord externe et cinq épines distales dont la deuxième est nettement plus allongée que les autres. Exopodite avec une épine apicale externe au premier article et trois épines apicales au troisième article.

P3 (fig. 4 c). Basis avec une épine externe et une rangée de spinules. Endopodite représenté par une mince épine. Exopodite massif avec plusieurs spinules au bord externe et deux fortes expensions chitinisées au bord interne ; l'apophyse se termine par une dent conique ; le pouce, lamelliforme, dépasse légèrement l'extrémité de l'exopodite.

P4 (fig. 4 d). Basis avec une longue épine externe. Endopodite avec une partie proximale élargie portant deux expansions chitineuses et une partie distale effilée dont le bord externe est orné de quatre dents. Exopodite avec une épine apicale au bord externe du premier article et deux épines apicales au troisième article ; bord interne du premier article avec deux spinules.

P5 (fig. 4 e, f). Très développée, elle se termine en pointe à l'angle distal interne. Le bord interne sub-rectiligne est armé de 5 à 7 spinules. Le bord externe très largement arrondi dans sa partie distale est armée de 3 épines. 


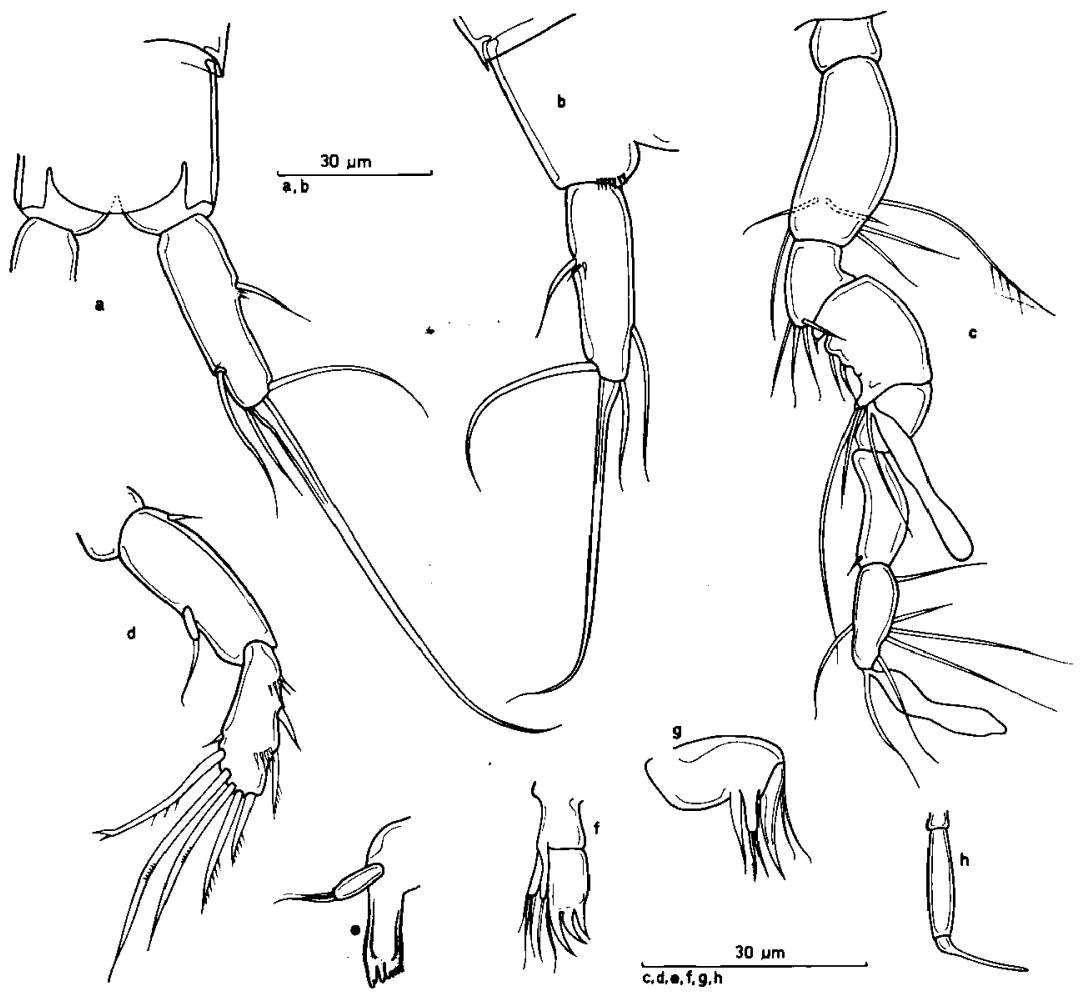

Fig. 3. Parastenocaris fontinalis meridionalis $\mathbf{n}$. s. sp., $\sigma$.

a : dernier segment abdominal et rame furcale, vue dorsale ; b : rame furcale, vue ventrale ; $:$ antennule ; : antenne ; $e:$ mandibule ; $f$ : maxillule ; $g$ : maxille $; h$ : maxillipède.

\section{- Femelle :}

Longueur moyenne du corps sans les soies furcales : $426 \mu \mathrm{m}$. Ornementation du corps, branches furcales et opercule anal comme chez le mâle.

Antennule (fig. 5 e) avec 7 articles. Antenne, mandibule, maxillule, maxille, maxillipède, P1 (fig. 5 a), P2 (fig. 5 b), et exopodite P4 (fig. 5 d) sans différences notables avec les appendices correspondants du mâle.

P3 (fig. 5 c). Basis avec une épine externe et une rangée de spinules. Exopodite biarticulé avec une épine distale externe au premier article et deux 


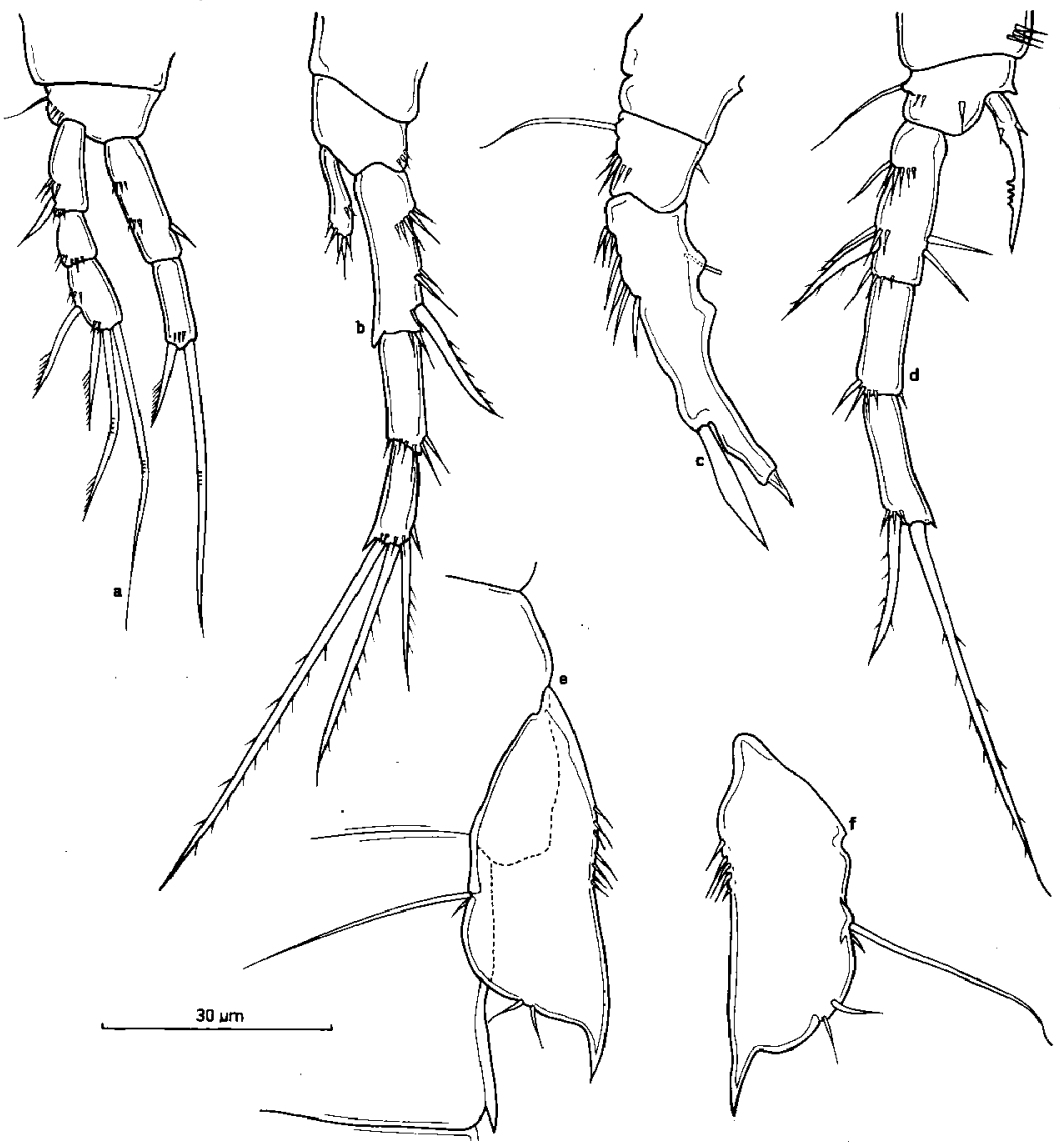

Fig. 4. Parastenocaris fontinalis meridionalis $\mathrm{n} . \mathrm{s} . \mathrm{sp} .$,

a : P1; b : P2 ; c : P3 ; d : P4; e : P5, vue latérale ; $:$ : 5, vue ventrale. 


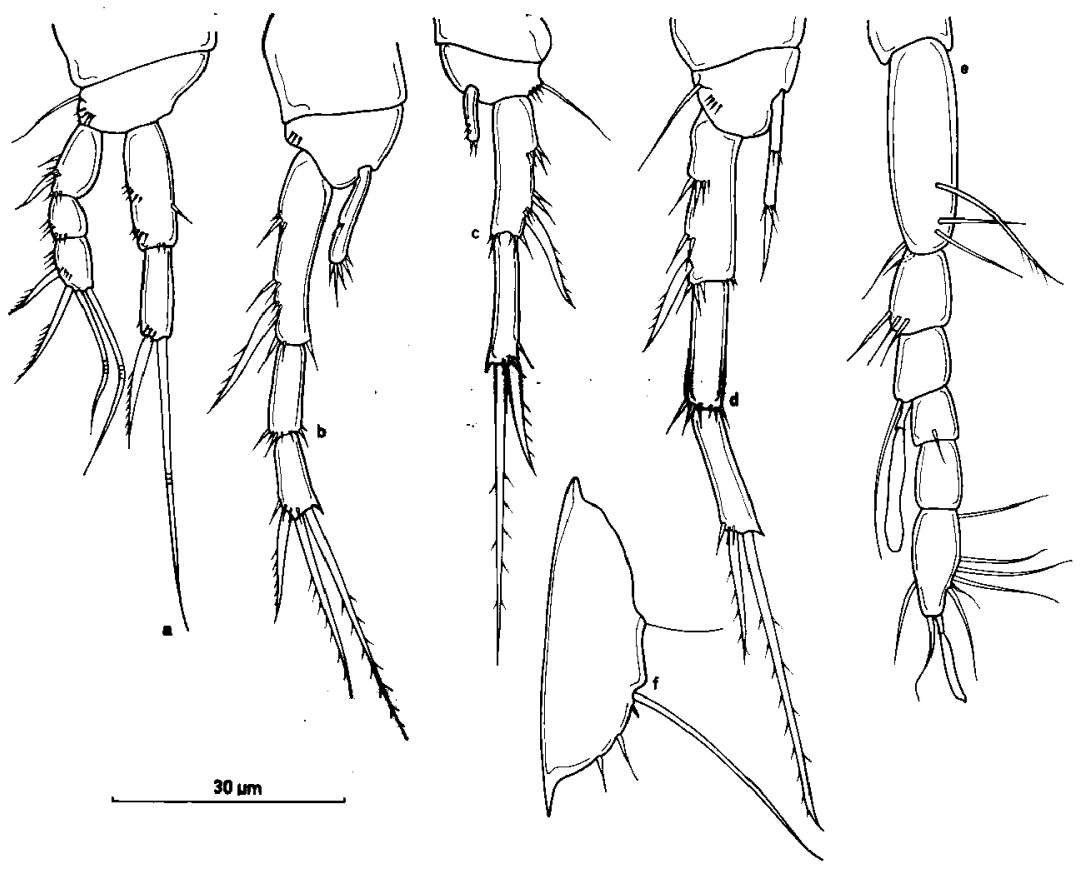

Fig, 5. Parastenocaris fontinalis meridionalis $\mathrm{n}, \mathrm{s} . \mathrm{sp}$. a : P1 ; b : P2 ; c : P3 ; d : P4 ; e : antennule ; f : P5.

épines apicales au second. Endopodite peu développé avec deux spinules au bord interne et deux spinules apicales.

Endopodite P4 (fig. 5 d). Digitiforme, il se termine par une forte épine à la base de laquelle s'insèrent trois spinules. Deux spinules ornent la partie proximale de l'endopodite.

P5 (fig. 5 f). De structure identique à celle du mâle, elle est toutefois moins développée avec un bord externe moins arrondi et un bord interne glabre.

\section{AFFINITES}

La structure des endopodites P2 et P4 des mâles provenant du Nert conduit à rattacher ces individus au groupe fontinalis (Lang 1948). C'est d'ailleurs avec l'espèce type de ce groupe, $P$. fontinalis Schnitter \& Chappuis 1915 qu'ils présentent le plus d'affinités. Les différentes populations de cette espèce montrent une assez grande variabilité notamment au niveau de la P5 des mâles. L'ornementation du bord interne de cette patte présente soit une expansion digitiforme plus 
ou moins développée (Schnitter \& Chappuis 1915, Kulhavy 1961) soit plusieurs soies (Hertzog 1938, Chappuis 1940, Kiefer 1959, Songeur 1961, Dussart 1966, Schminke - travail en cours). En 1960, Kiefer a crée la sous-espèce $P$. fontanalis borea ; elle se distingue de l'espèce type par la P5 du mâle dont le bord interne est armé d'une forte dent s'insérant audessus d'une lamelle hyaline. Les exemplaires décrits par Noodt en 1952 appartiendraient à cette sousespèce.

Mais, dans toutes ces populations, la forme générale de la $P 5$ du mâle reste identique : il s'agit d'une lamelle allongée, terminée en pointe et dont le bord externe distal est, tout au plus, faiblement convexe. La P5 du mâle de la forme du Nert s'écarte nettement de cette structure avec un bord distal externe très largement arrondi jusqu'au point d'insertion de la grande épine latérale.

Les autres caractères distinctifs des individus provenant du Nert sont les suivants :

- Branches furcales non rétrécies dans leur partie proximale.

- Opercule anal arrondi et non triangulaire.

- Pouce et apophyse de la P3 du mâle très développés par rapport à la partie proximale de l'exopodite.

- Endopodite P4 du mâle avec 2 excroissances seulement à l'extrémité distale de la partie renflée et quatre dents au bord externe de la partie effilée.

- P5 de la femelle sans ornementation au bord interne.

En revanche, les $\mathrm{P} 1$ et $\mathrm{P} 2$ des mâles et des femelles, les $\mathrm{P} 3$ et $\mathrm{P} 4$ des femelles présentent des structures pratiquement identiques à celles observées chez P. fontinalis.

A l'inverse de la plupart des espèces souterraines de Parastenocaris qui témoignent d'un endémisme élevé, $P$. fontinalis est caractérisée par une vaste distribution géographique. Curieusement, ce type de répartition est partagé par les autres espèces souterraines de Parastenocaris du nord de l'Europe occidentale comme $P$. vicesima, $P$. glacialis et $P$. phyllura (Enckell 1969). La capacité de ces espèces à vivre en eau interstitielle saumâtre ( $P$. vicesima, $P$. phyllura, $P$. fontinalis) ou à supporter le gel $(P$. glacialis) serait à l'origine de leur distribution actuelle (Enckell, op. cit.). $P$. fontinalis est la moins septentrionale d'entre elles puisqu'elle ne dépasse pas, au nord, la latitude du Danemark et du sud de la Suède. Récoltée dans de nombreuses stations de l'Allemagne de l'Ouest, elle a été également signalée aux Pays-Bas, en Allemagne de l'Est et en Tchécoslovaquie. Vers le sud, sa limite de répartition connue passe par le Boden-see (Kiefer 1959), Bâle (Schnitter \& Chappuis 1915), le lac Leman (Dussart 1966), le Rhône en amont de Lyon (Gibert et al 1977), pour remonter ensuite vers l'Alsace (Hertzog 1938) et la Lorraine (Songeur 1961). Sa présence dans les Pyrénées constitue donc une extension assez surprenante de son aire de répartition. La sousespèce borea a été récoltée dans le cours inférieur de la Weser, de l'Elbe, du Rhin, sur les bords de l'IJselmeers (Kiefer 1961) ainsi que dans le sud de la Suède. La sous-espèce meridionalis, outre le ruisseau du Nert, serait présente dans le Rhône (Schminke in litt.).

\section{Remerciements}

Je remercie A. Descouens qui a effectué le tri des récoltes et les dessins définitifs de cette note, A. Pitzalis, M. Bakalowicz et A. Mangin pour l'aide qu'ils m'ont apportée sur le terrain, $R$. Téchené qui a réalisé toute l'infrastructure technique de la station étudiée. Je remercie également $H$. K. Schminke qui m'a communiqué de précieux renseignements inedits sur $P$. fontinalis. F. Boineau a saisi le manuscrit.

\section{Travaux cités}

Chappuis (P.A.). 1925. - Sur les Copépodes et les Syncarides des eaux souterraines de Cluj et des monts Bihar. Bull. Soc. Sct. Cluj, $2: 157-182$.

Chappuis (P.A.). 1928. - Nouveaux Copépodes cavernicoles. Bull. Soc. Sci. Cluj, $4: 20-34$.

Chappuis (P.A.). 1936. - Subterrane Harpacticoiden aus Jugoslavien. Bull. Soc. Sci. Cluj, $8: 368-398$.

Chappuis (P.A.). 1937. - Subterrane Harpacticoiden aus NordSpanien. Bull. Soc. Cluj, $8: 556-571$.

Chappuis (P.A.). 1938. - Subterrane Harpacticoiden aus SüdItalien. Bull. Soc. Sci. Cluj, 9 : 153-183.

Chappuis (P.A.). 1940. - Die Harpacticoiden des Grundwassers des unteren Maintales. Arch. F. Hydrobiol., $36: 286-305$.

Chappuis (P.A.). 1955. - Notes sur les Copépodes. 18. Nouveaux Harpacticoides des Pyrénées, 19. Harpacticoïdes cavernicoles de Grèce, 20. Copépodes Harpacticoides des Iles du Pacifique. Notes Biospéol, $10: 89-101$.

Chappuis (P.A.) \& Rouch (R.). 1959, - Harpacticoïdes cavernicoles des Basses-Pyrénées. Ann. Spéléol., $14: 197-211$.

Cottarelli (V.). 1969. - Nuove Parastenocaris (Copepoda, Harpacticoida) dell'Italia centro-meridionale. Riv. Idrobiol., 8 : 1-28.

Cottarelli (V.). 1972. - Parastenocaris (Copepoda, Harpacticoida) di alcuni laghi vulcanici del Lazio. Ist. Lomb. Sc. Lett., $106: 138-155$. 
Cottareli (V.) et Drigo (E.). 1972. - Sulla presenza di Parastenocaris orcina Chappuis (Cop. Harpacticoida) in acque interstiziali del lago di Bracciano. Notiz. Circ. Speleol, Rom., 1-2 ; $51-54$.

Cottarelli (V.), Saporito (P.E.) \& Puccetti (A.). 1981. - Parastenocaris stellae $\mathrm{n}$. sp. della falda iporreica del fiume Cedrino (Sardegna). Frag- entom., Roma, $16: 1.7$.

Dussart (B.). 1966. - Copépodes de la faure benthique du Léman. Vie et Milieu, $17: 283-302$.

Enckell (P.H.). 1965. - New Harpacticoids from Spain. Acta Univ. Lund., $19: 3-9$.

Enckell (P.H.). 1969. - Distribution and dispersal of Parastenocarididae (Copepoda) in northern Europe. Oikos, 20 : 493-507.

Gibert (J.), Ginet (R.), Mathieu (J.), Reygrobellet (J.L.) \& SeyedReihani (A.). 1977. - Structure et fonctionnement des écosystèmes du Haut-Rhône français. IV. Le peuplement des eaux phréatiques ; premiers résultats. Annls Limnol., 13 : 83-97.

Gourbault (N.) \& Lescher-Moutoué (F.). 1967. - Sur la faune hypogée peuplant le sous-ecoulement d'une rivière de moyenne altitude. C.R. Acad. Sc. Paris, 241: 1813-1816.

Gourbault (N.) \& Lescher-Moutoué (F.). 1968. - Etude de la faune hypogée peuplant le sous-ècoulement du Nert. $A n n$. Spéléol., 23, $4: 735-742$.

Hertzog (L.). 1938. - Crustaceen aus unterirdischen Biotopen des Rheintales bei Straßburg. Zool, Anz., 123 ; 45-56.

Kiefer (F.). 1959. Unterirdisch lęende Ruderfußkrebse vorn Hochrhein u. Bodensee. Beitr. naturkundl. Forschg. Südwestdeutschl., $18: 45-52$.

Kiefer (F.). 1960. - Psammobionte Ruderfußkrebse (Crust. Cop.) aus dem Gebiet der Unterweser und von der Insel Helgoland. Zool. Anz., 165 : 30-37.
Kiefer (F.). 1961 - Über einige Parastenocariden (Copepoda Harpacticoida) aus den Niederlanden. Crustaceana, 3 : 115-119.

Kulhavy (V.). 1961. - Uber das Vorkommen der west-und osteuropaischen Elemente in der Crustaceenfauna der böhmischen unterirdischen Gewässer. Acsa soc. zool. bohemosiov., 25 : 297-301.

Lang (K.). 1948. - Monographie der Harpacticiden. 2 vol. Lund. $1682 \mathrm{p}$.

Noodt (W.). 1952. - Subterrane Copepoden aus Norddeutschland. Zool. Anz., 148: 331-343.

Noodt (W.). 1955. - Limnisch-subterrane Harpacticoiden (Crust. Cop.) aus Norditalien. Zool. Anz., 154 : 78-85.

Pesce (G.L.), Galassi (D.P.) \& Cottarelli (V.). 1988. - First representative of the family Parastenocarididae from Sicily (Italy), and description of two new species of Parastenocaris Kessler (Crustacea, Copepoda : Harpacticoida). Bull. zool. Mus. Univ. Amsterdam, 11:137-142.

Rouch (R.). 1987. - Copépodes Harpacticoïdes stygobies d'Algérie. Bijar. Dierk., $57: 71-86$.

Rouch (R.). 1988. - Parastenocaris vandeli n. sp., nouvel Copépode Harpacticide psammique des Pyrénées. Crustaceana, 54, $2: 163-170$.

Schnitter (H.) \& Chappuis (P.A.). 1915. - Parastenocaris fontinalis nov. spec., ein neuer Süßwasserharpacticide. Zool. Anz. $45:$ : 290-302.

Songeur (M.). 1961. - Un nouveau Copépode psammique de Lorraine. Vie et Milieu, $12: 483-496$. 